\title{
Efficiency of ensuring the survivability of logistics information and control systems
}

\author{
Vladimir Anisimov ${ }^{1, *}$, Evgeniy Anisimov ${ }^{2}$, and Tatyana Saurenko ${ }^{2}$ \\ ${ }^{1}$ Peter the Great St. Petersburg Polytechnic University (SPbPU), Graduate School of Business and \\ Management, Polytechnicheskaya, 29, St. Petersburg, 195251, Russia \\ ${ }^{2}$ Peoples Friendship University of Russia (RUDN University), 6 Miklukho-Maklaya St, Moscow, \\ 117198, Russia
}

\begin{abstract}
The article proposes a methodological approach to assessing the effectiveness of measures to ensure the survivability of management systems for logistics business processes. The effectiveness of these measures is determined as the degree of preservation of the capabilities of control systems to perform their functions under the conditions of destructive influences. It is proposed to use a function as an indicator of effectiveness, the argument of which is the change in the duration of the control cycle associated with the need to restore the operability of the control system after destructive influences. The proposed approach forms the basis for constructing mathematical models of decision support for the choice of means and methods of ensuring survivability for a wide class of management systems for logistic business processes.
\end{abstract}

\section{Introduction}

The distributed nature of the production and consumption of raw materials, components and finished products determines the increasing influence of the quality of logistics business processes on the competitiveness of companies in the markets for goods and services. In modern conditions, one of the most promising areas for improving the quality of logistic business processes is the digital transformation of information support for decision making, which allows you to quickly process all the information available at all stages of planning and management [1 - 9]. At the same time, an increase in the volume of processed information, the introduction of information technology in the management of logistics business processes is accompanied by a significant increase in the vulnerability of the respective information management systems from various destructive influences [10 - 18]. Therefore, maintaining the survivability of logistics information and control systems under the conditions of such impacts is becoming one of the important problems of digitalization in the field of managing logistics business processes. Maintaining their survivability is associated with the need for appropriate organizational and technical measures. A wide range of such events, their various effectiveness and cost make it necessary to choose the most effective of them. Such a choice implies the presence of an appropriate methodological apparatus.

${ }^{*}$ Corresponding author: an-33@yandex.ru 
The development of a methodological approach to assessing the effectiveness of survivability measures for the management of logistic business processes is the goal of this article.

\section{Description of the approach}

The management of logistic business processes in the technological aspect can be represented as a process of formation and subsequent implementation of control actions [19 -21]. When the performance indicator is mathematically represented as a function, the argument of which is the change in the duration of the management cycle associated with the need to restore the operability of the logistics business process management system, the following assumptions are correct.

1. If the control cycle $t$ does not exceed some predetermined value $T$, then the capabilities of the system are realized in full.

2. On the interval $\mathrm{T}<\mathrm{t}<\mathrm{Tcr}$, the degree of realization of opportunities gradually decreases.

3. At $t>$ Tcr, the control process is disrupted.

Then the dependence of the degree of implementation of the capabilities of the logistics business process on the duration of the management cycle can be represented by the ratio:

$$
W(t)=\left\{\begin{array}{c}
1, t \leq T \\
e^{-\lambda(t-T)}, t>T
\end{array},\right.
$$

where $\mathrm{W}(\mathrm{t})$ - the degree of implementation of the capabilities of the logistic business process from the duration of its management cycle;

$\lambda$ - the rate of decrease in the degree of realization of potential opportunities of the business process if the duration of the management cycle exceeds the value of $T$.

Without loss of generality, we can also assume that in the absence of destructive influences, the duration $t$ of the control cycle is T. In the presence of destructive influences, the value of $t$ exceeds the nominal duration $\mathrm{T}$ of the control cycle by the time ${ }^{\tau}$ necessary to eliminate their negative consequences, that is, $t=T+\tau$. Therefore, relation (1) takes the form:

$$
W(\tau)=e^{-\lambda \tau}
$$

The parameter $\lambda$ in the general case depends on the time $\tau$, i.e., $\lambda=\lambda(\tau)$.

Assuming that the elimination of negative consequences begins immediately after each destructive impact, and the time spent on elimination is random and significantly less than the time interval between successive actions, a random value $\tau_{\text {can be represented as: }}$

$$
\tau=\sum_{r=1}^{R} \tau_{r},
$$

where $\mathrm{R}$ is the number of destructive influences during the control cycle;

$\tau_{r}$ - time to eliminate the consequences of the $\mathrm{r}-\mathrm{th}(\mathrm{r}=1,2, \ldots, \mathrm{R})$ destructive impact.

It is also natural to assume that the destructive influences themselves constitute a random stream of events. Then the value of $R$ in relation (3) is also random. 
Based on the assumption that random variables $\tau_{r}(\mathrm{r}=1,2, \ldots, \mathrm{R})$ are independent of each other, distributed according to one law and do not depend on $\mathrm{R}$, we use the apparatus of characteristic functions to construct the distribution function of a random variable $\tau$.

Let $\mathrm{q}(\mathrm{x})$ denote the distribution density function of random variables

$\tau_{r}(\mathrm{r}=1,2, \ldots, \mathrm{R})$. Then the corresponding characteristic function takes the form:

$$
\varphi(z)=\int_{-\infty}^{\infty} e^{i z x} q(x) d x
$$

where $i=\sqrt{-1}$ is the imaginary unit;

$\mathrm{z}$ is a real number.

If we denote by $\mathrm{pR}$ the probability that $\mathrm{R}$ destructive actions will occur during the control cycle, then, based on the multiplicative property, we obtain that the characteristic function $\xi(z)$ of the random time $\tau$ of counteraction to these actions is determined by the relation:

$$
\xi(z)=\sum_{R=0}^{\infty} p_{R}[\varphi(z)]^{R}
$$

Given relation (5), the density $\mathrm{g}(\mathrm{x})$ of the distribution of a random variable $\tau$ can be represented as:

$$
g(x)=\frac{1}{2 \pi} \int_{-\infty}^{\infty} e^{-i z x} \sum_{R=0}^{\infty} p_{R}[\varphi(z)]^{R} d z
$$

Given the finite nature of the quantities

$$
\sum_{R=0}^{\infty} p_{R}[\varphi(z)]^{R} \leq \sum_{R=0}^{\infty} p_{R}[\varphi(0)]^{R}<\infty
$$

in relation (6), we can replace the order of integration and summation. Then we obtain:

$$
g(x)=\sum_{R=0}^{\infty} p_{R} \frac{1}{2 \pi} \int_{-\infty}^{\infty} e^{-i z x}[\varphi(z)]^{R} d z
$$

Taking into account relation (8), the mathematical expectation $\tau^{*}$ of the time for eliminating the consequences of destructive effects is equal to:

$$
\tau^{*}=\int_{0}^{\infty} x\left[\sum_{R=0}^{\infty} p_{R} \frac{1}{2 \pi} \int_{-\infty}^{\infty} e^{-i z x}[\varphi(z)]^{R} d z\right] d x=\sum_{R=0}^{\infty} p_{R} \int_{0}^{\infty} x\left[\frac{1}{2 \pi} \int_{-\infty}^{\infty} e^{-i z x}[\varphi(z)]^{R} d z\right] d x
$$

Substituting the value of $\tau^{*}$ in (2), we obtain the ratio for determining the average degree of realization of the potential capabilities of the logistic information management system in the current conditions:

$$
W\left(\tau^{*}\right)=\exp \left\{-\lambda \sum_{R=0}^{\infty} p_{R} \int_{0}^{\infty} x\left[\frac{1}{2 \pi} \int_{-\infty}^{\infty} e^{-i z x}[\varphi(z)]^{R} d z\right] d x\right\} .
$$

For a constructive presentation of relation (10), it is necessary to determine: 
the intensity of reducing the degree of realization of the potential opportunities of a particular logistic business process in the interval $\mathrm{T}<\mathrm{t}<\operatorname{Tcr}$ (parameter $\lambda$ );

distribution function of the flow of destructive influences (function $p_{R}$ );

density distribution of the time to eliminate the consequences of each of them (function $\mathrm{q}(\mathrm{x}))$.

These parameters are determined based on statistical information about the corresponding logistic business process.

\section{Conclusions}

On the whole, the experience in managing logistic business processes of enterprises in the real sector of the economy indicates that in the context of the digitalization of the economy, their control systems become very vulnerable to various deliberate and unintended destructive influences. Therefore, ensuring the survivability of these systems is becoming one of the important problems. The complexity of solving this problem and significant material and reputation losses from miscalculations when making decisions on the implementation of appropriate tools and methods for ensuring the survivability of control systems at all stages of their life cycle necessitates a comprehensive justification of these decisions. The tool for this justification is the assessment of effectiveness. The methodological approach proposed in the article to the mathematical formalization of the problem of evaluating the effectiveness of measures to ensure the survivability of control systems is based on fairly general characteristics of the systems under study and logistic business processes. In this regard, it can serve as a theoretical basis for constructing specific models and methods for supporting decision-making on the introduction of tools and methods to ensure the survivability of the considered management systems for logistic business processes.

\section{References}

1. A.V. Tepikin, Logistics (Moscow, 2012)

2. E.G. Anisimov, T.N. Saurenko, M.R. Gapov, E.S. Rodionova, The model for determining rational inventory in occasional demand supply chains, International Journal of Supply Chain Management 8, 1, 86-89 (2019)

3. D. Waters, Logistics. An Introduction to Supply Chain Management (USA: JohnWileyandSons, 2015)

4. V.G. Anisimov, P.D. Zegzhda, A.F. Suprun, E.G. Anisimov, The problem of innovative development of information security systems in the transport sector, Automatic Control and Computer Sciences 52, 8, 1105-1110 (2018)

5. V.V. Shcherbakov, A.V. Merzlyak, E.O. Koskur-Ogly, Automation of business processes in logistics (Saint-Petersburg, 2016)

6. V.G. Anisimov, E.G. Anisimov, T.N. Saurenko, M.A. Sonkin, The model and the planning method of volume and variety assessment of innovative products in an industrial enterprise, J. Phys.: Conf. Ser. 803(1), 012006 (2017) DOI: 10.1088/17426596/803/1/012006

7. V.S. Lukinskiy, V.V. Lukinskiy, N.G. Pletnev, Logistics and supply chain management (Moscow: Yurait, 2016) 
8. V. Anisimov, A. Chernysh, E. Anisimov, Model and algorithm for substantiating solutions for organization of high-rise construction project, E3S Web of Conferences P. 03003 (2018)

9. L.B. Mirotin, Fundamentals of engineering logistics in transport (Moscow, 2012)

10. V. Anisimov, E. Anisimov, M. Sonkin, A resource-and-time method to optimize the performance of several interrelated operations, International Journal of Applied Engineering Research 10, 17, 38127-38132 (2015)

11. Yu.N. Ivanova, Methodology of strategic planning of Russian TRANS-regional corporations (Moscow, 2016)

12. I. Zaychenko, N. Grashchenko, V. Anisimov, T. Saurenko, E. Anisimov, V. Zhigulin, Models for predicting damage due to accidents at energy objects and in energy systems of enterprises, E3S Web of Conferences 02041 (2019)

13. T. Saurenko, E. Anisimov, V. Anisimov, A. Levina, Comparing investment projects of innovative developing strategies of municipalities, based on a set of indicators, MATEC Web of Conferences 01038 (2018)

14. O.V. Yudakova, Role of logistics strategy in strategic planning of enterprises, Scientific forum 6, 345 (2017)

15. E.G. Anisimov, V.G. Anisimov, M.A. Sonkin, Mathematical simulation of adaptive allocation of discrete resources. In: Proceedings of the 2016 Conference on Information Technologies in Science, Management, Social Sphere and Medicine, ITSMSSM 2016. ACSR: Advances in Computer Science Research 282-285 (2016)

16. P. Du, L. Xu, Q. Chen, S. Tsai, Pricing competition on innovative product between innovator and entrant imitator facing strategic customers, International J. of Production Research 1-19 (2016)

17. T. Saurenko, V. Anisimov, E. Anisimov, A. Smolenskiy, N. Grashchenko, Justification of financing of measures to prevent accidents in power systems, Advances in Intelligent Systems and Computing 983, 295-305 (2019)

18. A.A. Moiseenko, The role of logistics strategy in the activities of transport enterprises, Economics and economic Sciences 6, 39 (2015)

19. T. Saurenko, E. Anisimov, V. Anisimov, I. Bagaeva, Conceptual provisions for assessing the effectiveness of energy saving, E3S Web of Conferences 02066 (2019)

20. V.G. Anisimov, P.D. Zegzhda, E.G. Anisimov, T.N. Saurenko, S.P. Prisyazhnyuk, Indices of the effectiveness of information protection in an information interaction system for controlling complex distributed organizational objects, Automatic Control and Computer Sciences 51, 8, 824-828 (2017)

21. V.G. Anisimov, P.D. Zegzhda, E.G. Anisimov, D.A. Bazhin, A risk-oriented approach to the control arrangement of security protection subsystems of information systems, Automatic Control and Computer Sciences 50, 8, 717-721 (2016) 